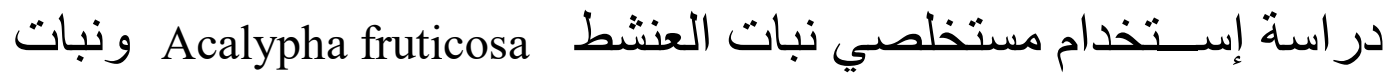

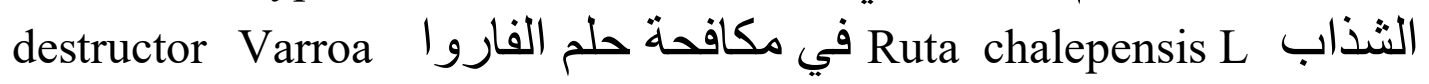

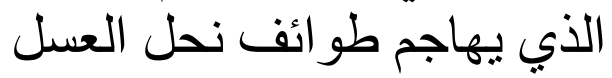

$$
\text { كلية الزرال عبده أحمد الشرعبي وأحمد علي راشد الجمالي البيطري - جامعة إب - البين }
$$

المستخلص، أجريت هذه الدراسة في منحل المعهد المهني الزراعي ـ العدين ـ محافظة إب ـ الجمهورية اليمنية على طو ائف نحل من السلالة اليمنية Apis mellifera jemenitica مرباة في خلايا حديثة (لانجستروث) وتم

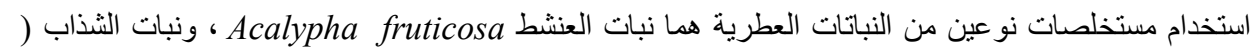

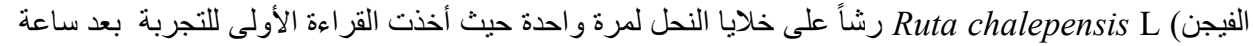
من التطبيق والقر اءة الأخرى بعد 24 ساعة من التطبيق ولمدة 6 أيام متتالية ، أجريت التجارب مابين الساعة التهات

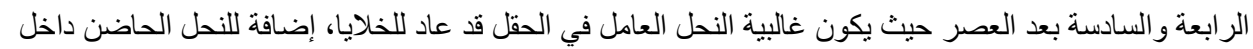

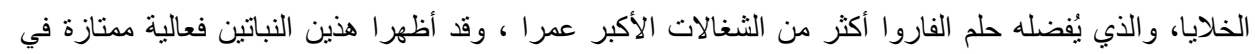

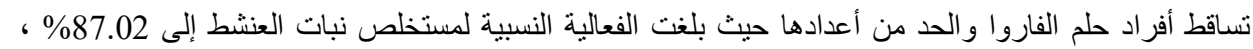

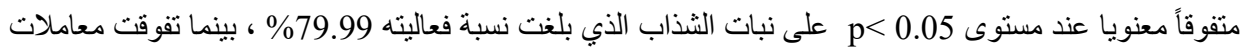

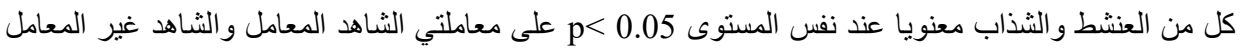

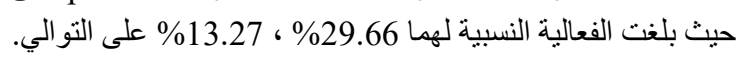

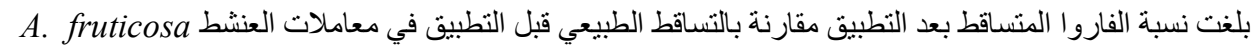

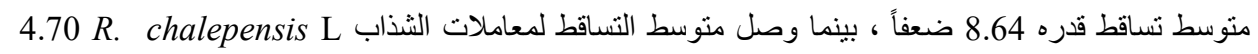

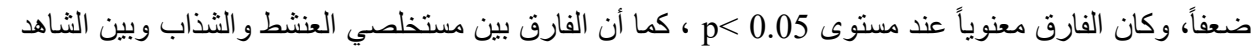

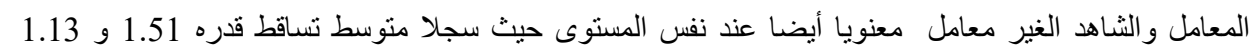

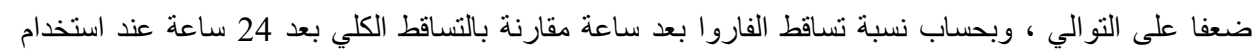

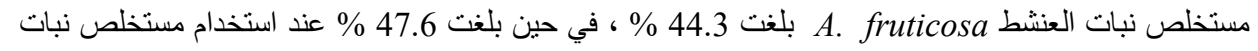

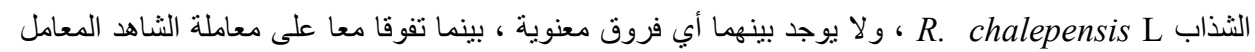

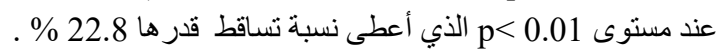

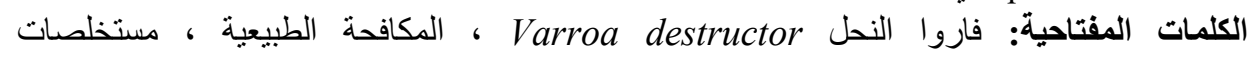
نباتية، Ruta chalepensis L ، Acalypha fruticosa

انه يقلل من قدرة الطائفة على تلقيح النباتات

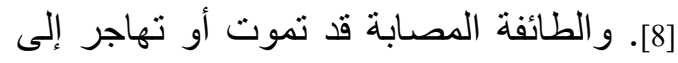

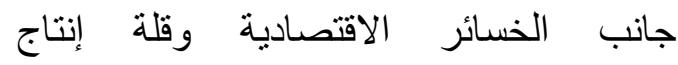
Varroa العسل.[14] ويُعتبر حلم الفاروا destructor العسل،حيث يسبب تدهور مجتمع النحل في الطي

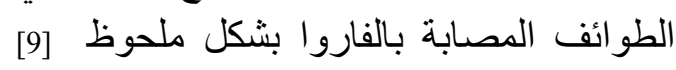
ويعد طفيل الفاروا العائق الرئيس أمام تطور لئن الفور

\section{المقدمة}

حلم الفاروا Varroa destructor من أخطر

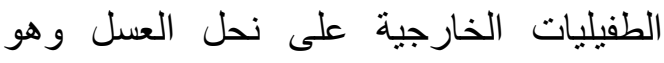
موضع قلق لمربي النحل في جميع أنحاء العالم حيث يتغذى على دم النحل Haemolymph من أفراد الحضنة والنحل الكبير مما يسبب دمل صليب

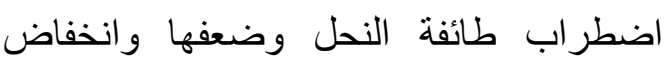
الحضنة ونتوه النحل الغير بالغ والكبير، كما وضعا وانها 
استخدام تلك المركبات الكيميائية واسع الانتشار

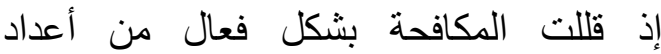

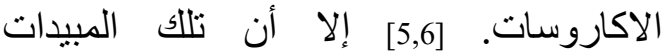
الأكاروسية Acaricides أظهرت العات العديد من المساوئ على الرغم من فعاليتها العالية في

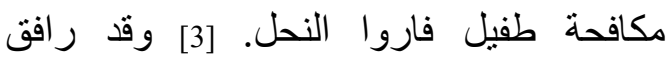

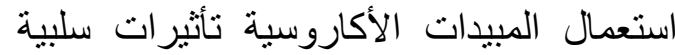

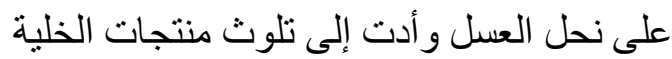

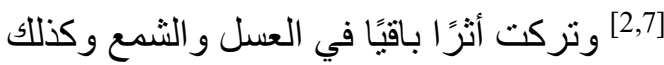
في البروبوليس.[12] و هنالك مشكلة متز ايدة في في الكئ المستقبل القريب تتمثل في مقاومة الطفيل للمبيدات الأكاروسية وظهور سلالات مقاومة. [13]و انخفاض الحساسية للمبيدات الأكاروسية من قبل الطفيليات من خلال الاستخدام المتكرر

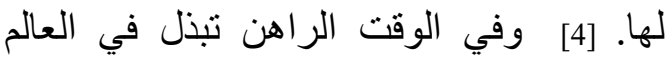
جهودا كبيرة بحثًا عن مو اد جديدة في الدكافحة،

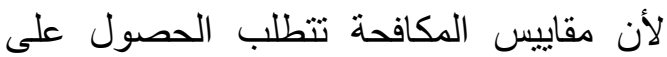

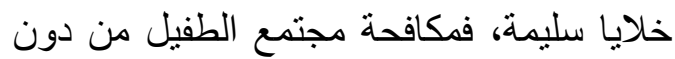
تطبيقات كيميائية هو اختيار آمن بيئيًا وبالإمكان حدوثه عمليًا باستخدام المواد الطبيعية.

\section{الهذف من الارسه}

تهدف هذه الدراسة إلى اختبار كفاءة مستخلصات بعض النباتات العطرية في مكافحة فاروا النحل بستعاض بها عن استخدام المكافحة

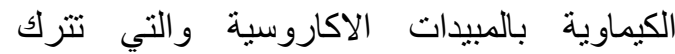
متنقيات تؤثر على صحة الإنسان و البيئة، ومن أجل الحصول على منتجات نحلية طبيعية خالية من الاندان الآثار المتبقية للمبيدات والمواد الصناعية أيضا.

(التغطية النحلية حوالي 6 أقراص) ، (شكل1) وزودت بقواعد خاصة لعد الفاروا المتساقطة screened bottom board ودهنت بالفازلين (شكل2) واستخدم نوعين من المستخلصات النباتية هما:

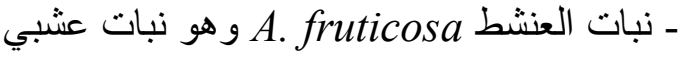
بري ذو رائحة عطرية غالبا ما يستخدم محليا في نيات
تربية النحل في الوقت الراهن، إذ تمنص أنثى

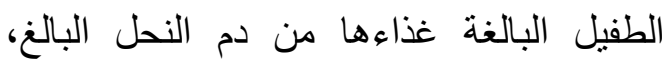

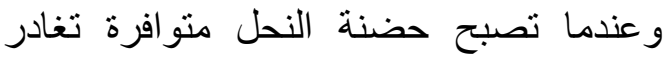
الطفيليات النحل البالغ لتتكاثر داخل نخاريب النغي الحضنة متغذية على دمها، و عندما يخرج النحل النحل

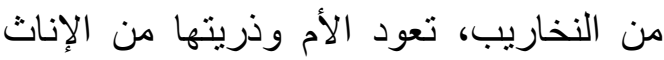

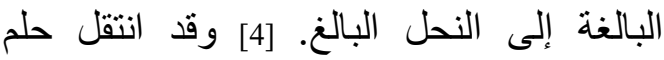
الفاروا إلى اليمن عبر الحدود الجنوبية الغربية

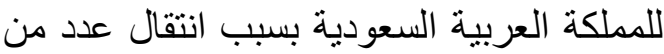
النحالين اليمنيين مع خلاياهم إلى حدود الميلى الكلكة

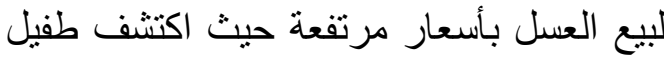

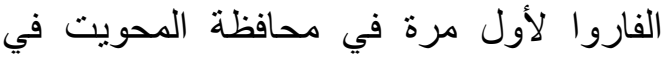
أغسطس عام 1990. [11] بالإضافة إلى تأثير هذا الطفيل المباشر في النحل وحضنته والتغذِّي

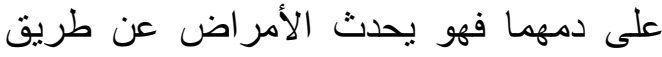

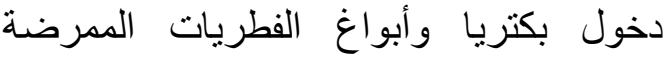

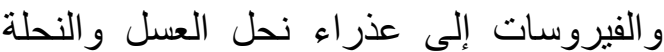
البالغة، كما ينقل العدوى إلى العذراء العئ السليمة

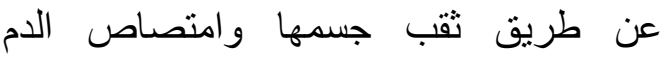

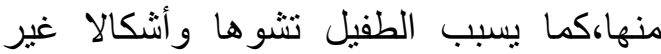
طبيعية في النحل البالغ،وانخفاضيًا في الوزن.

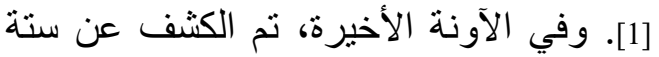

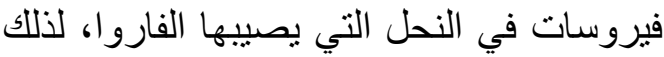

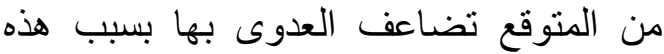

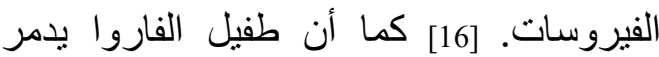

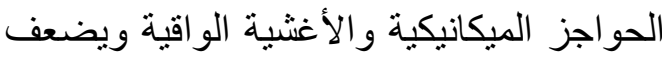
الجهاز المناعي للنحل. [10] تم استعمال العديد من المركبات الكيميائية في معظم أنحاء العالم ضد الفاروا، وأصبح

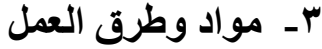

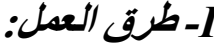

أجريت هذه التجربة في منحل المعهة المهني الزراعي في منطقة العدين - محافظة إب -

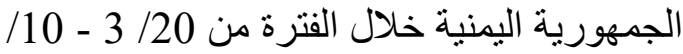

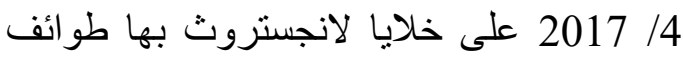
نحل منوسطة القوة وبدرجات منساوية تقريبا 
مرشات بدوية ـ ميزان حساس - عدسات تكبير

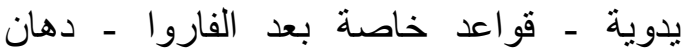
الفازلين ، نم اختيار 16 خلية بطريقة عشو ائية،

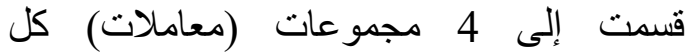
مجمو عة تتكون من 4 خلايا نحل وضعت علت عليها علامات توضح كل مجموعة (معاملة) على تلى على على على

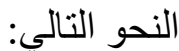

المجبوعة الأولى: استخدم فيها مستخلص نبات A. fruticosa العنشط المجموعة الثانية: استخدم فيها مستخلص نبات R. chalepensis الثذاب المجبوعة الثالثة: الثـاهد معامل بالماء المحتوي على 5\% سكر.

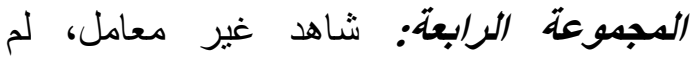
يستخدم فيه أي شي. تحضير المستخلصات النباتية: تم إتباع طريقة الاستخلاص الساخن للنباتات: ـ النقيع في العاء المغلحي (مستحلب): تم وزن

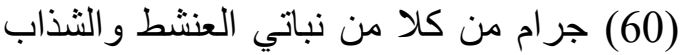

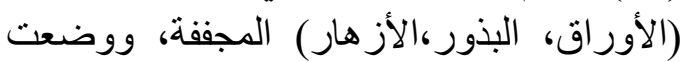
كلاً على حدة في أوعية من الأستانلس ستيل ولنيل

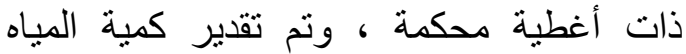
اللازمة لتغطية المادة النباتية لكلا النباتين ووضعت في إناء معدني على النار حتى درجة لإنه

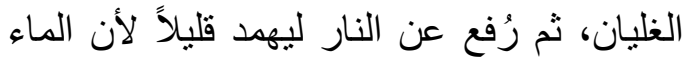

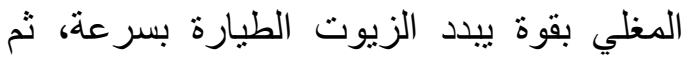
يسكب الماء الساخن فوق الجزء النباتي وغلق

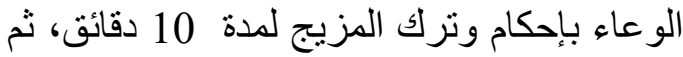
تمت عملية التصفية بواسطة قطع من قماش

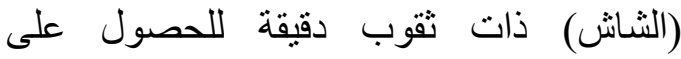
المستخلص أو الخلاصة. بعد الحصول على المستخلص أضيف إليه سكرً، وتم تعبئتها في أوان زجاجية معتمة لحين
الطب التقليدي لعلاج أمر اض الحساسية الجلدية

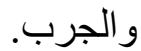

R. chalepensis L نبات الثذاب (الفيجن) وهي شجيرة دائمة الخضرة ينبعث منها رائحة عطرية قوية يستعمل الفيجن في علاج التهابات الأذن و الأزمات الصدرية وصعوبة بلة التنفس. حيث جُمع النباتان من منطقة العدين - محافظة

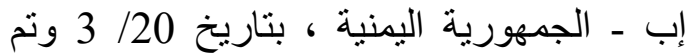

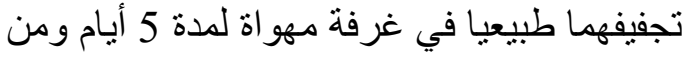
ثم تجزئتها بواسطة خلاط كهربائي إلى قطع صغيرة من السيقان والأوراق والأزهار الجافة.
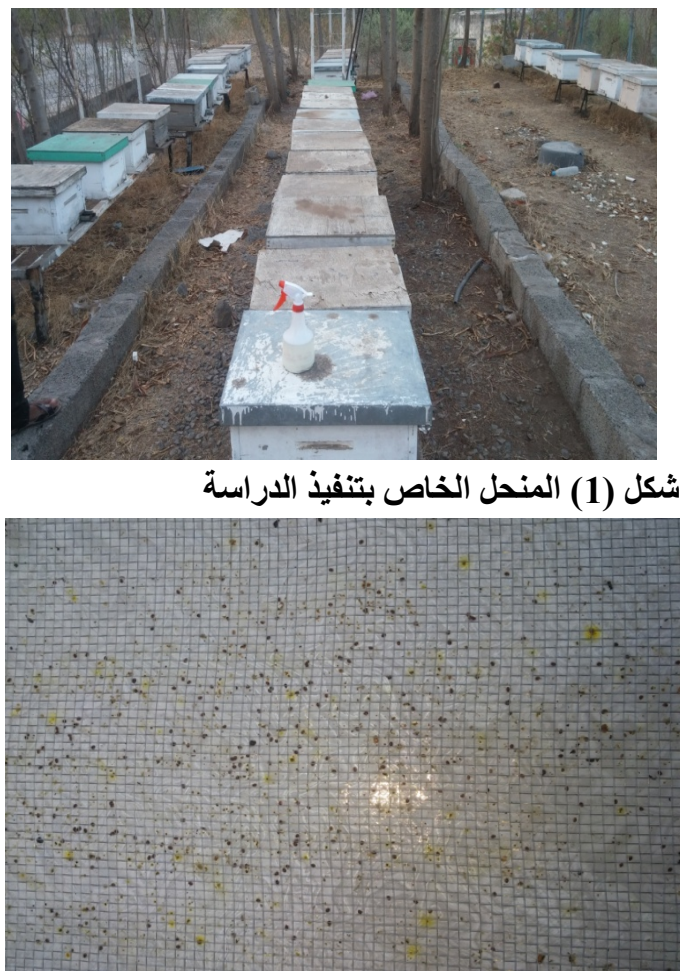

شكل (2) قواعد عد الفاروا المتساقطة

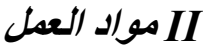
استعملت بعض الأدوات في التجربة منل: 


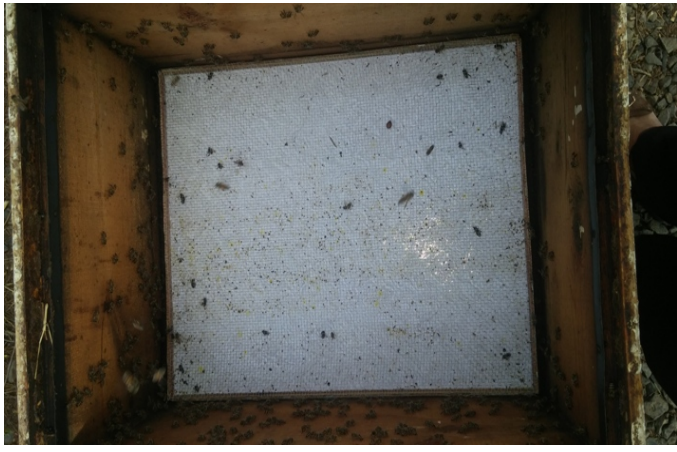

شكل (4) قواعد عد الفاروا المتساقطة

وقد نم أخذ القراءة الأولى للفاروا المتساقط على القو اعد بعد ساعة من التطبيق، ثم أخذت القرة القداءة الثانية بعد 24 ساعة من التطبيق لمدة 6 أيام

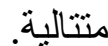
حساب متوسط الكوت الطبيعي للفاروا : تم أخذ قراءة التساقط الطبيعي للفاروا الميت طبيعيًا لمدة 3 أيام قبل المعالجة، من أجل حساب

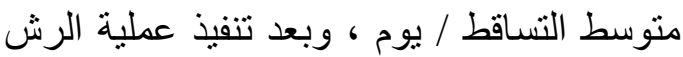

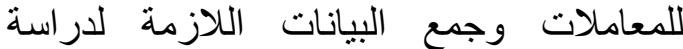

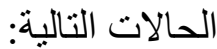

أولا: حساب فعالية المادة النباتية في تساقط الاتط

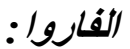
تؤخذ قر اعة تساقط الفاروا الميت بعد ساعة من

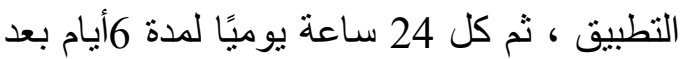
المعالجة، وتحسب الفعالية النسبية بعد كل 24 ساعة من التطبيق، حتى تلاشي تأثثر المادة النباتية ، وقد تم حساب الفعالية النسبية في قتل نلئل الفاروا في خلايا التجربة والثـاهد بتطبيق المعادلة التالية: - المالية الفاعلية النسبية = متوسط التساقط قبل المعالجة ــونوسط التساقط بعد المعالجة / متوسط التساقط

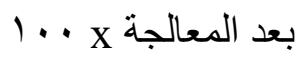

بدء التطبيق، وفي اليوم التالي وضعت

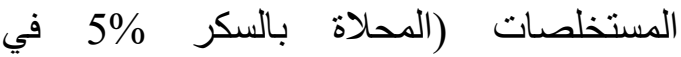
البخاخات ) مرشات صغيرة حيث استخدم لكل

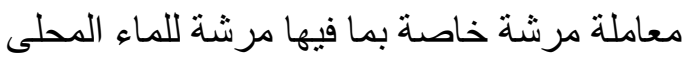
بالسكر 5\% ، المستخدمة في مكررات الثـاهد

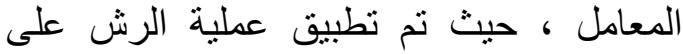
خلايا المعاملات بعد وضع قواعد عد الفاروا

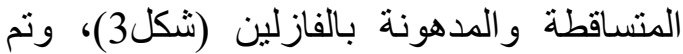
الرش بطريقة منتظمة على كل وجه 3 رشات لكل قرص من أقر اص الخلية وذللك بحسب كثافة النحل،(شكل4) وتمت تغطية الخلايا بإحكام.

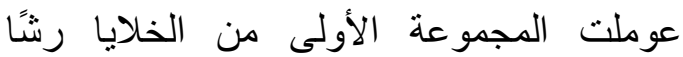
بمستخلص نبات العنشط تركيز 5 \%

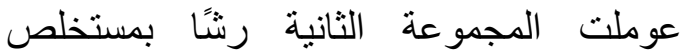

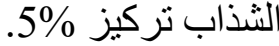
كما عوملت المجموعة الثالثة رشاً بالماء المحتوي 5\% سكر (شاهد معامل).

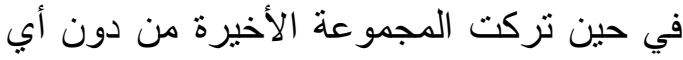
معاملة (شاهد غير معامل).

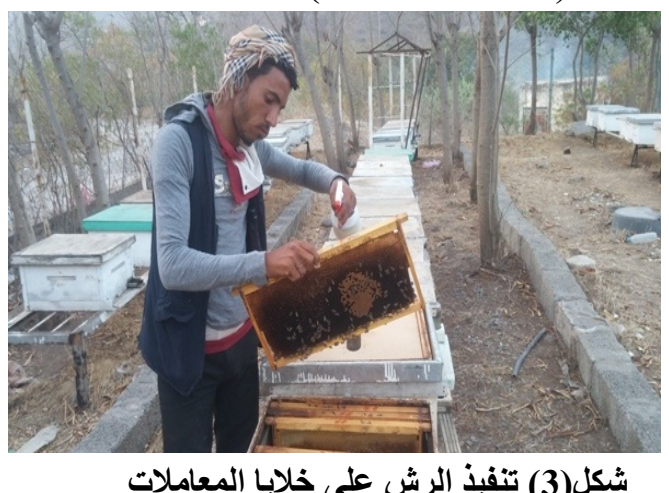
شكل(3) تنفيذ الرش على خلايا المعاملات 
عدد الفاروا المتساقط بعد ساعة من المكافحة /

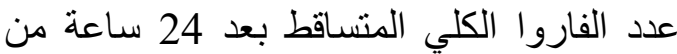
100 x المكافحة حلات النتائج إحصائياً باستخدام اختبار F في one way اتجاه واحد كامل العشوائية completely randomized المتوسطات بإجراء اختبار أقل فرق P = على مستوى احتمالية L.S.D بنوية ${ }^{[15]}$.0.01, $\mathrm{P}=0.05$ النتائج

أظهرت نتائج التحليل الإحصائي وجود فارق معنوي على مستوى 0.05 > بين كل من العنشط والثذاب، بينما كان الفارق معنويا بين كل من من بن مستخلص نباتي العنشط و الثذاب وبين منابن الثناهد المعامل و الثاهد الغير معامل ، و عند مقارنة الفعالية النسبية لمتوسط تساقط الفارو ا بخلايا الثـاهد المعامل بالماء تركيز 5\% سكر، و الشاهد غير المعامل كانت

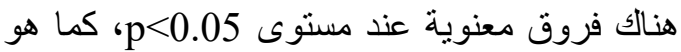
موضح في الثكل (5) ويعزى زيادة التساقط للشاهد المعامل بسبب السلوك ألتنظيفي للنحل داخل الخلايا أثناء التطبيق نظر ا للرش بالماء تركيز 5\% سكر.
ثانباً:النسبة بين التساقط بعد التطبيق(ضعف): وهي عدد أضعاف الفاروا المتساقطة بعد 24 ساعة من إجراء المكافحة مقارنة بمتوسط التساقط الطبيعي قبل المكافحة

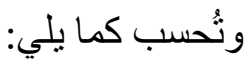
النسبة بين التساقط الطبيعي بعد وقبل التطبيق عدد الفاروا المتساقط بعد 24ساعة من المكافحة / متوسط التساقط الطبيعي قبل المكافحة. ثالثاً: نسبة الفاروا المتساقطة بعد ساعة مقارنة بالتساقط الكلي بعد 24ساعة. ويتم حسابها بتطبيث المعادلة التالية: أولا- حساب الكفاعة النسبية: تشير نتائج الجدول (1) أن استخدام مستخلص نباتي العنشط Acalypha fruticosa و الثذاب Ruta chalepensis L مكافحة حلم الفاروا رشاً، أعطت فعالية عالية في تساقط حلم الفاروا Varroa destructor من أفراد

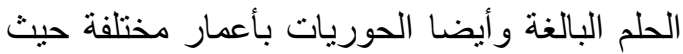

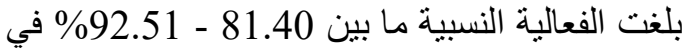
الخلايا (المكررات) VI‘II وبمتوسط فعالية نسبية قدر ها 87.02\% لمستخلص نبات العنش و 77.2183.25 في الخلايا III ، II وبمتوسط فعالية نسبية بلغت 79.99\% لمستخلص نبات الثذاب، بينما بلغت نسبة الفعالية النسبية في خلايا الثاهد المعامل \%29.66، و الثاهد غير المعامل 13.27\%، وقد

جدول (1) تأثير مستخلص نبات العنشط Acalypha fruticosa ونبات الثذاب Ruta chalepensis L

على مكافحة حلم الفاروا Varroa destructor 



\begin{tabular}{|c|c|c|c|c|c|c|c|c|c|c|c|c|}
\hline \multirow{2}{*}{ 承 } & \multirow{2}{*}{ 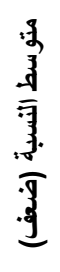 } & \multirow{2}{*}{ التبد وقبلة بلنيل } & \multirow{2}{*}{ 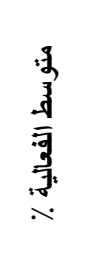 } & \multirow{2}{*}{ 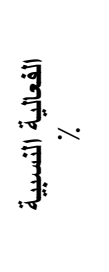 } & \multirow{2}{*}{$\begin{array}{l}\overline{3} \\
3 \\
3 \\
3\end{array}$} & \multirow{2}{*}{ 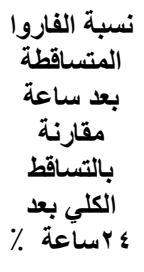 } & \multicolumn{2}{|c|}{ التطبسق افظط بعدا } & \multirow{2}{*}{ 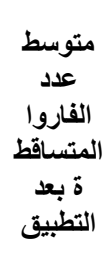 } & \multirow{2}{*}{ اللفاريبع } & \multirow{2}{*}{ 亨 } & \multirow{2}{*}{ 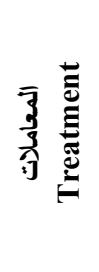 } \\
\hline & & & & & & & س بعاعة & سأعة & & & & \\
\hline \multirow{4}{*}{$\mathbf{a}$} & \multirow{4}{*}{$\begin{array}{c}8.6 \\
4\end{array}$} & 9.06 & \multirow{4}{*}{87.02} & 88.96 & \multirow{4}{*}{44.3} & 42.7 & 42.2 & 18 & 42.2 & 4.66 & I & \multirow{4}{*}{ 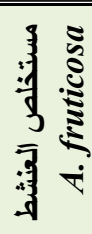 } \\
\hline & & 5.38 & & 81.40 & & 46.5 & 43 & 20 & 43 & 8 & II & \\
\hline & & 6.76 & & 85.21 & & 44.4 & 51.8 & 23 & 51.8 & 7.66 & III & \\
\hline & & 13.36 & & 92.51 & & 43.5 & 71.2 & 31 & 71.2 & 5.33 & VI & \\
\hline \multirow{4}{*}{ b } & \multirow{4}{*}{$\begin{array}{c}4.7 \\
0\end{array}$} & 5.4 & \multirow{4}{*}{79.99} & 81.48 & \multirow{4}{*}{47.6} & 48.2 & 27 & 13 & 27 & 5 & I & \multirow{4}{*}{ 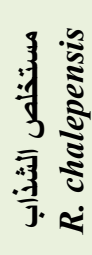 } \\
\hline & & 5.97 & & 83.25 & & 41.9 & 59.7 & 25 & 59.7 & 10 & II & \\
\hline & & 4.39 & & 77.21 & & 51.5 & 40.8 & 21 & 40.8 & 9.3 & III & \\
\hline & & 4.56 & & 78.05 & & 48.8 & 28.7 & 14 & 28.7 & 6.3 & VI & \\
\hline \multirow{4}{*}{ c } & \multirow{4}{*}{$\begin{array}{c}1.4 \\
2\end{array}$} & 1.89 & \multirow{4}{*}{29.66} & 49.6 & \multirow{4}{*}{22.8} & 16 & 12.5 & 2 & 12.5 & 6.3 & I & \multirow{4}{*}{ 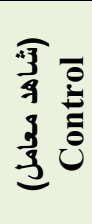 } \\
\hline & & 1.23 & & 18.69 & & 32.5 & 12.3 & 4 & 12.3 & 10 & II & \\
\hline & & 1.92 & & 47.83 & & 26.1 & 11.5 & 3 & 11.5 & 6 & III & \\
\hline & & 1.03 & & 2.50 & & 16.7 & 12 & 2 & 12 & 11.7 & VI & \\
\hline \multirow{4}{*}{ c } & \multirow{4}{*}{$\begin{array}{c}1.1 \\
3\end{array}$} & 1.08 & \multirow{4}{*}{13.27} & 7.62 & \multirow{4}{*}{ - } & - & 10.5 & - & 10.5 & 9.7 & I & \multirow{4}{*}{ 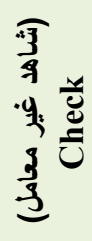 } \\
\hline & & 0.89 & & $\mathbf{0}$ & & - & 9.8 & - & 9.8 & 11 & II & \\
\hline & & 1.75 & & 42.73 & & - & 11 & - & 11 & 6.3 & III & \\
\hline & & 1.03 & & 2.73 & & - & 11 & - & 11 & 10.7 & VI & \\
\hline
\end{tabular}

الأرقام التي تحمل أحرف متثابهة لا تختلف معنويأ عثد مستوى

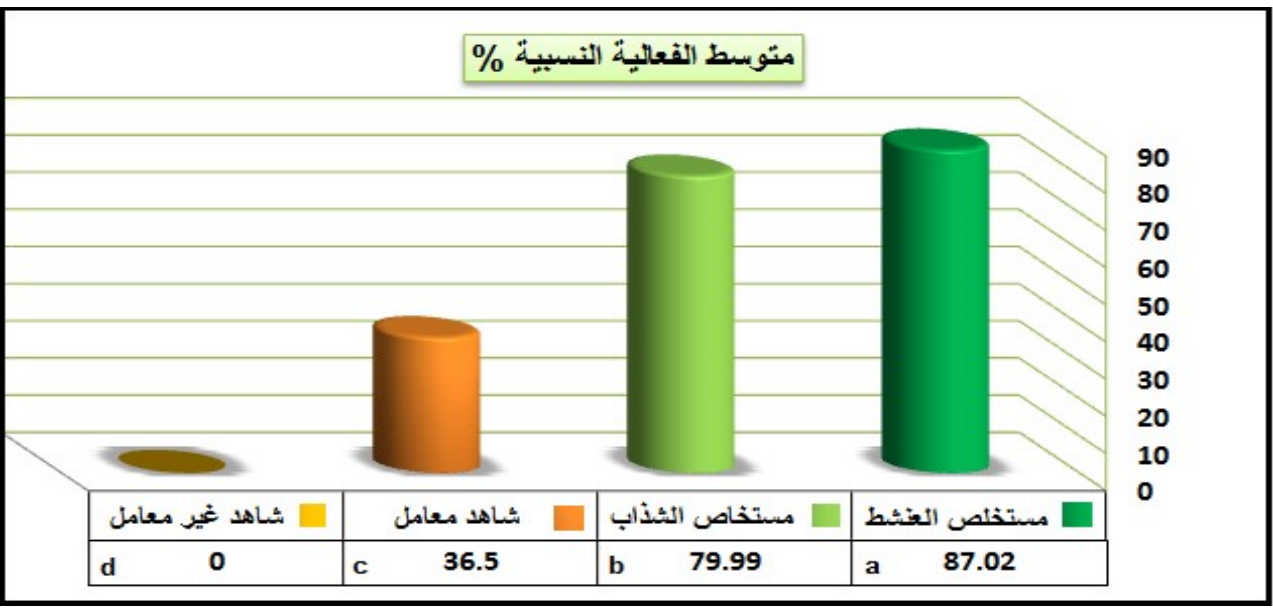

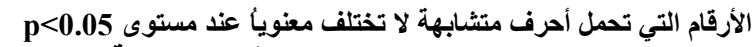

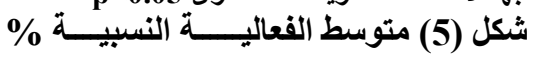



بين 4.39 و5.97 وبمتوسط قدره 4.70 ضعفاً بالنسبة للنساقط قبل التطبيق عند استخدام مستخلص

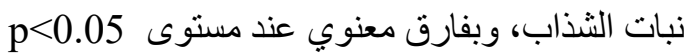

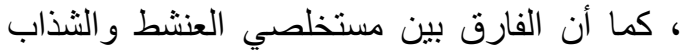

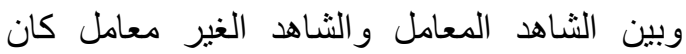

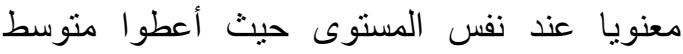
تساقط قدره 1.51 و 1.13 على التو الي، شكل (6).
ثانياً- النسبة بين التساقط بعد وقبل التطبيق (ضعف): أظهرت النتائج في الجدول (1) أيضاً أن النسبة بين

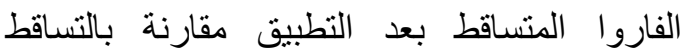

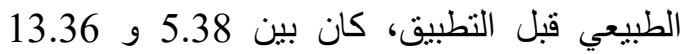

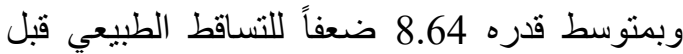
التطبيق عند استخدام مستخلص العنشط ، بينما كانت

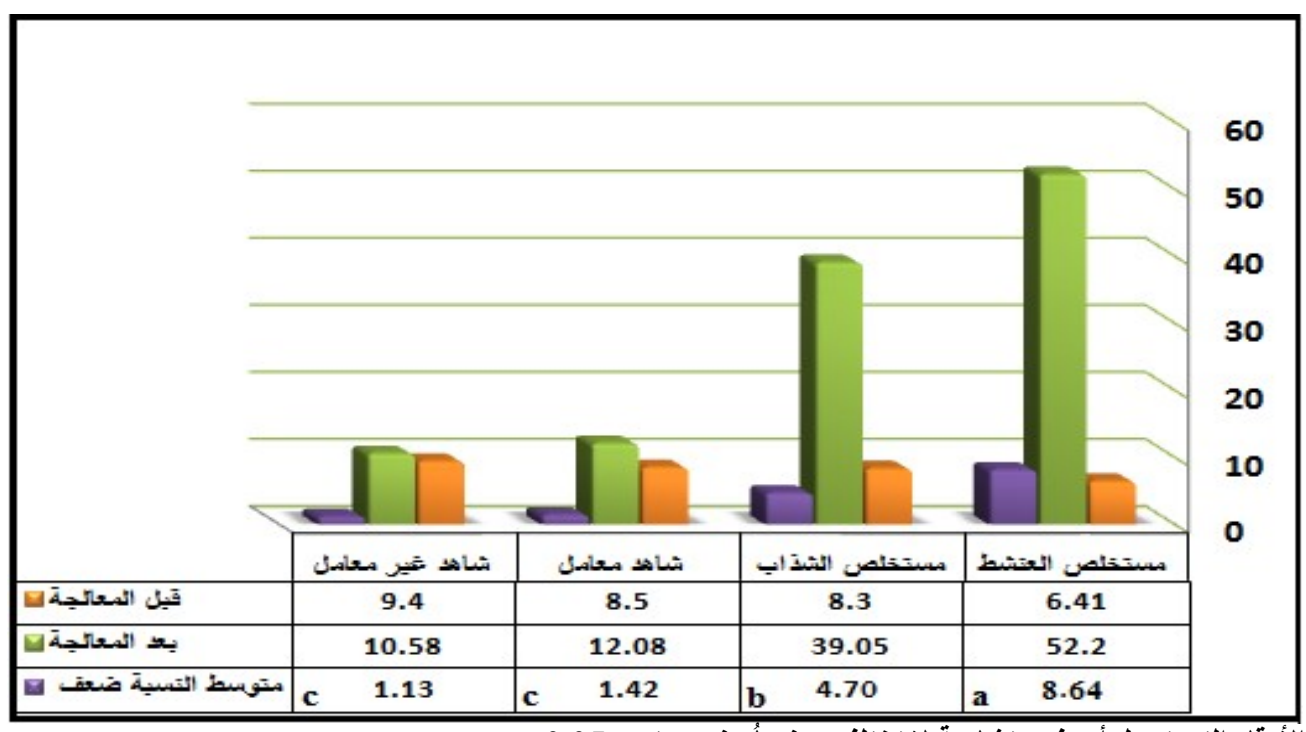

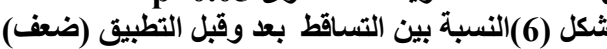

نبات الثذاب تفوق بسيط بمتوسط 3.3\% عن مستخلص نبات العنشط ناتج عن تأثنير قوة الرائحة

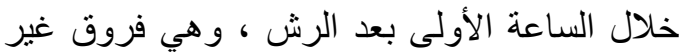

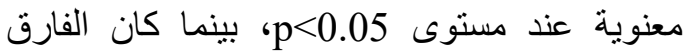
معنويا عند نفس المستوى بين كل من مستخلصي

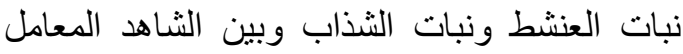
الذي أعطى متوسط نسبة تساقط قدرها 22.8 \% م

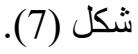

ثالثاً نسبة الفاروا المتساقطة بعد ساعة مقارنة بالتساقط الكلي بعد 24ساعة: بالعودة للجدول (1) نلاحظ أن منوسط تساقط الفاروا بعد ساعة مقارنة بالتساقط الكلي بعد 24 ساعة عند استخدام مستخلص نبات بلف العنشط Acalypha fruticosa بلغت 47.6 \% عند استخدام مستخلص نبات الثذاب

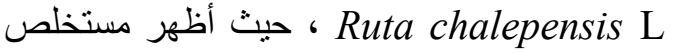





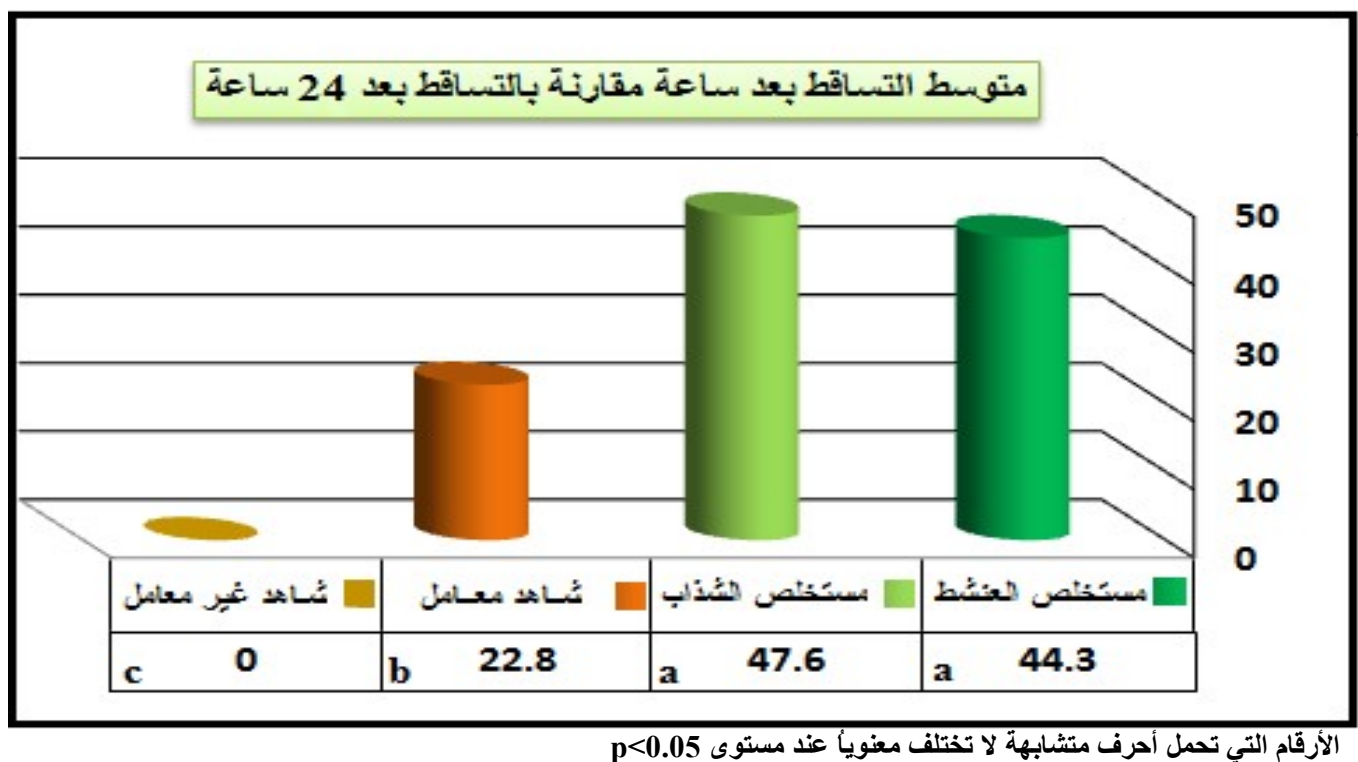

شكل (7) متوسط نسبة تساقط الفاروا بعد ساعة مقارنة بالتساقط الكلي بعد 24 ساعة \%

[5] Calis J. N. M., W. J. Boot, and J. Beetsma. Model evaluation of methods for Varroa jacobsoni mite control based on trapping in honey bee brood. Apidologie 30: 197-207. (1999 b).

[6] Daher-Hjaij, N. and Alburaki, A. Control of Varroa jacobsoni Oud. by fumigation with natural plant substances, Arab J. Pl. Prot., 24: 93-97. (2006).

[7] De Guzman L., T. Rinderer, G. Delatte, and R. Macchiavelli. Varroa jacobsoni Oud emans tolerance in selected stocks of Apis mellifera L. Apidologie 27: 193-210. (1996).

[8] De Jong, D.; Goncalves, L. S. and Morse R. A. Dependence on climate of the virulence of Varroa jacobsoni. Bee World, 65 (3): 117-121. (1984).

[9] Figen K., Girisgin, A. O. Aydin, L. Varroacidal efficacies of essential oils extracted from Lavandula officinalis,

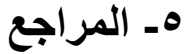

[1] Ball B. V. Secondary infections and diseases associated with Varroa jacobsoni. In: the Varroosis in the Mediterranean region, CIHEAM, Paris (France) 21: 49-58. (1996).

[2] Ben-Hamida. T. Chemotherapy against Varroa jacobsoni: Efficiency and side effects. In the Varroosis in the Mediterranean Region, CIHEAM, Paris (France) 21: 77- 86. (1997).

[3] Branco M., N. C. Kidd, and R. Pickard. Development of Varroa jacobsoni in colonies of Apis mellifera iberica. in Mediterranean climate. Apidologie, 30: 491- 503. (1999).

[4] Calis J. N. M. I. Fries, and S. Ryrie. Population modeling of Varroa jacobsoni Oud. Apidologie 30: 111124. (1999 a). 
mites in honey bee colonies. 14-18. (1999).

[13] Milani N., and G. Della Vedova. Determination of LC50 in the mite Varroa jacobsoni of the active substances in Perizin and Cekafix. Apidologie 27 : 175-184 (1996).

[14] Needham, G. R. Status report on Varroa jacobsoni. Am., Bee J., 128: 106- 110 (1988).

[15] SAS Institute. JMP version 7.0.1.SAS Institute Inc, Cary, NC.USA, (2007).

[16] Tentcheva, D.; Gauthier L.; Zappulla N.;Dainat, B.; Cousserans, F.; Colin, M. E. and Bergoin ,M, Prevalence and seasonal variations of six bee viruses in Apis mellifera L. and Varroa destructor mite populations in France. Appl. Environ. Microbiol.,70: 7185-7191, . (2004).
Foeniculum vulgare, and Laurus nobilis in naturally infested honeybee (Apis mellifera L.) colonies. Turk. J. Vet. Anim. Sci. 2012; 36(5): 554-559. (2012).

[10] Glinski, Z. The effect of Varroa jacobsoni Oud. on the incidence and cause of chalkbrood disease in Apis mellifera L. colonies. Rev. Agric. Entom., 079-09747 (1991).

[11] Hoppe H. Vergleichende untersuchungen Zur biotechnischen Bekämpfung der Var- Roatose, Dissertation, Justus-Liebig-universität Giessen (1990).

[12] Imdorf A., and S. Bogdanov. Use of essential oils for the control of Varroa jacobsoni. Swiss Apicultural Institute. Coordination in Europe of research on integrated control of Varroa 


\title{
Study Application of Acalypha Fruticosa and Ruta Chalepensis Plant Extracts Against Varroa Mite, Varroa Destructor Attacking Honeybee Colonies
}

\author{
Gamal A. A. Alsharabi and Ahmed Ali Rashed Aljamali \\ Faculty of Agriculture and Veterinary Medicine - University of Ibb, Yemen
}

\begin{abstract}
This study was conducted at the Institute of Agricultural Vocational Apiary Training in Odin- Ibb- Republic of Yemen, on the bee cultivars of the Yemeni strain Apis mellifera jemenitica in modern hives (Langstroth). Extracts of the aromatic plants, Acalypha fruticosa and Ruta chalepensis were tested against Varroa mite, Varroa destructor attacking honeybee colonies by spraying honeybee trays. The falling mites were counted after one hour and 24 hours of application, then daily for 6 consecutive days. The experiments were conducted from $4 \mathrm{pm}$ and $6 \mathrm{pm}$, where the majority of field workers had returned to the cells, In addition to the incubator workers inside the cells, which is infested by varroa more than the older workers. The obtained results showed that both of tested extracts highly reduced varroa mites, moreover, there were significant differences among all treatments at $\mathrm{p}<0.05$ compared to control. The percentages numbers of fallen mites were 47.6, 44.3, and $22.8 \%$ for $R$. chalepensis, A. fruticosa, and control, respectively. The relative efficacy against varroa mites of $A$. fruticosa extract was $87.02 \%$, and $R$. chalepensis was $79.99 \%$, while it was $29.66 \%$ and $13.27 \%$, for control and check treatments, respectively. In the case of the varroa that fell after the application compared to the normal fall before application (twice) in the treatment of Acalypha fruticosa high at an average of 8.64 (weakness). The mean variability of Ruta chalepensis $\mathrm{L}$ treated hives was 4.70 (weakness), with a significant difference at $\mathrm{p}<0.05$. The difference was also significant between $A$. fruticosa and $R$. chalepensis $\mathrm{L}$ and treated control colonies and in non-controlled (control). Comparing the relative folds of reduction efficacy, it was 8.64, 4.70, and 1.42 folds for $A$. fruticosa, $R$. chalepensis, and control, respectively.
\end{abstract}

